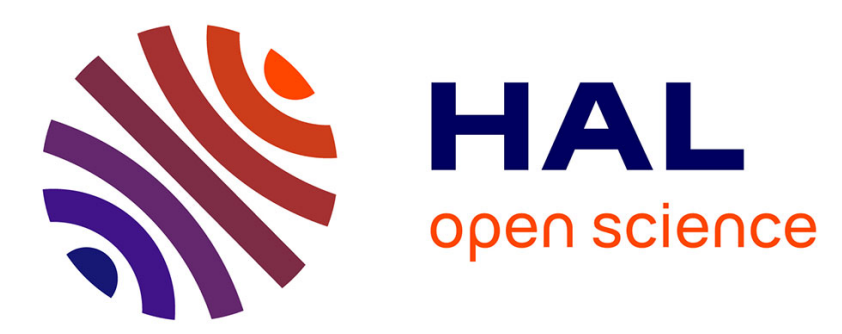

\title{
Molecular dynamics simulations of aluminium diffusion in decagonal quasicrystals
}

\author{
Stephen Hocker, Franz Gähler, Peter Brommer
}

\section{To cite this version:}

Stephen Hocker, Franz Gähler, Peter Brommer. Molecular dynamics simulations of aluminium diffusion in decagonal quasicrystals. Philosophical Magazine, 2006, 86 (06-08), pp.1051-1057. 10.1080/14786430500259734 . hal-00513580

\section{HAL Id: hal-00513580 \\ https://hal.science/hal-00513580}

Submitted on 1 Sep 2010

HAL is a multi-disciplinary open access archive for the deposit and dissemination of scientific research documents, whether they are published or not. The documents may come from teaching and research institutions in France or abroad, or from public or private research centers.
L'archive ouverte pluridisciplinaire HAL, est destinée au dépôt et à la diffusion de documents scientifiques de niveau recherche, publiés ou non, émanant des établissements d'enseignement et de recherche français ou étrangers, des laboratoires publics ou privés. 


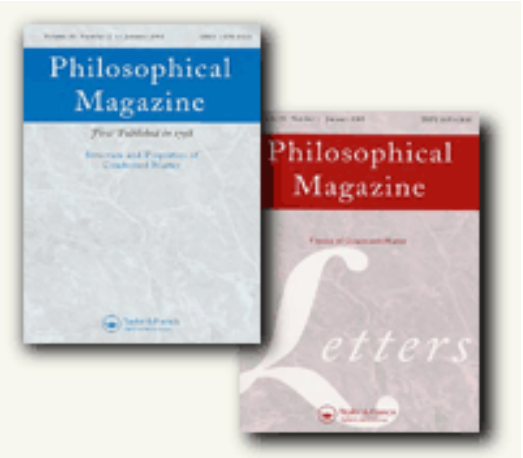

\section{Molecular dynamics simulations of aluminium diffusion in decagonal quasicrystals}

\begin{tabular}{|c|c|}
\hline Journal: & Philosophical Magazine \& Philosophical Magazine Letters \\
\hline Manuscript ID: & TPHM-05-May-0196.R1 \\
\hline Journal Selection: & Philosophical Magazine \\
\hline $\begin{array}{r}\text { Date Submitted by the } \\
\text { Author: }\end{array}$ & 13-Jul-2005 \\
\hline Complete List of Authors: & $\begin{array}{l}\text { Hocker, Stephen; Universität Stuttgart, Institut für Theoretische } \\
\text { und Angewandte Physik } \\
\text { Gähler, Franz; Universität Stuttgart, Theoretische und Angewandte } \\
\text { Physik } \\
\text { Brommer, Peter; Universität Stuttgart, Institut für Theoretische und } \\
\text { Angewandte Physik }\end{array}$ \\
\hline Keywords: & quasicrystals, molecular dynamic simulations, diffusion \\
\hline Keywords (user supplied): & \\
\hline
\end{tabular}

\section{(5) SholaroNE \\ Manuscript Central}




\title{
Molecular dynamics simulation of aluminium diffusion in decagonal quasicrystals
}

\author{
Stephen Hocker, Franz Gähler, and Peter Brommer \\ Institut für Theoretische und Angewandte Physik \\ Universität Stuttgart
}

July 13, 2005

\begin{abstract}
Aluminium diffusion in decagonal $\mathrm{Al}-\mathrm{Ni}-\mathrm{Co}$ and $\mathrm{Al}-\mathrm{Cu}-\mathrm{Co}$ quasicrystals is investigated by molecular dynamics simulations. Results obtained with newly developed EAM potentials are compared to previous work with effective pair potentials [Phys. Rev. Lett. 93, 075901 (2004)]. With both types of potential, strong aluminium diffusion is observed above two thirds of the melting temperature, and the general behaviour of the system is quite similar. The diffusion constant is measured as a function of temperature and pressure, and the activation enthalpies and activation volumes are determined from the resulting Arrhenius plot. For a number of important diffusion processes, the energy barriers are determined with molecular statics simulations. The qualitative behaviour of the dynamics is also confirmed by ab-initio simulations.
\end{abstract}

Keywords: Diffusion; Molecular dynamics simulation; Quasicrystals

\section{Introduction}

Atomic diffusion in quasicrystals is essential for many physical processes in these complex alloys. It is required for the formation of the equilibrium phase during high temperature annealing and for the motion of dislocations and other defects. Unfortunately, due to the lack of suitable radiotracers it is very hard to measure $\mathrm{Al}$ diffusion experimentally. No such studies have been presented so far. In a recent paper [1] we have shown that molecular dynamics (MD) simulations can complement the experimental methods. Molecular dynamics not only allows to determine the microscopic dynamics of the $\mathrm{Al}$ atoms, but also to measure the macroscopic diffusion constant as a function of temperature and pressure. MD simulations thus provide an excellent tool for the study of atomic dynamics both at the microscopic and the macroscopic level.

The quality of MD simulations crucially depends on the potentials. As quantummechanical simulations are limited to a few hundred atoms, which can neither provide 
enough statistics nor model the aperiodic nature of quasicrystals, classical effective potentials have to be used [1]. Since central pair potentials show some defiencies [2] in the description of metals and intermetallic compounds, we choose so-called Embedded Atom Method (EAM) [3] or glue potentials [2], which are better suited for metals. These include an additional embedding term, taking the form

$$
V=\frac{1}{2} \sum_{i \neq j} \Phi_{i j}\left(r_{i j}\right)+\sum_{i} U_{i}\left(n_{i}\right), \quad n_{i}=\sum_{i \neq j} \rho_{j}\left(r_{i j}\right),
$$

where $U_{i}$ describes the energy of embedding atom $i$ in a density $n_{i}$, which is the sum of contributions $\rho_{j}$ from neighbours $j$ at distances $r_{i j}$. We developed EAM potentials for Al-Ni-Co and Al-Cu-Co using the Force Matching Method of Ercolessi and Adams [4]. For a selection of small reference configurations the cohesion energies, stresses and forces on individual atoms are calculated by quantum-mechanical ab-initio methods. All ab-initio calculations in this work are carried out with VASP [5-7], using the Projector Augmented Wave (PAW) method [8]. The potential parameters are then fitted to optimally reproduce these data, using a least square fit. Details on the potential developement are given in [9]. The capabilities of the obtained potentials crucially depend on the selection of reference configurations. For the present purpose (diffusion simulations), high temperature structures are given a large weight in the collection of reference configurations, so that the potentials are trained to describe high temperature processes well.

Three different model structures are considered. The Ni-rich structure of decagonal AlNi-Co (composition $\mathrm{Al}_{72} \mathrm{Ni}_{21} \mathrm{Co}_{9}$ ) and the structure of decagonal Al-Cu-Co (composition $\left.\mathrm{Al}_{70} \mathrm{Cu}_{10} \mathrm{Co}_{20}\right)$ both consist of an alternating stacking of two different layers decorating the same hexagon-boat-star (HBS) tiling, which results in a period of about $4 \AA[10,11]$. We use sligthly modified variants determined in relaxation simulations, where it was found that the two innermost $\mathrm{Al}$ atoms in the star tiles prefer different positions, and also break the $4 \AA$ periodicity locally to an $8 \AA$ periodicity [1]. The model structure for $\mathrm{Al}-\mathrm{Cu}-\mathrm{Co}$ is obtained from the Ni-rich decoration by replacing each Ni-Ni pair on supertile edges by a $\mathrm{Cu}$-Co pair. Lacking a detailed quasiperiodic model of the Co-rich phase, simulations of this structure were performed with a periodic approximant [12]. Its structure differs significantly from the Co-rich sample used in [1].

Insert Fig. 1 about here

\section{Results}

In previous work [1] with pair potentials, significant diffusion of $\mathrm{Al}$ was observed in $\mathrm{Ni}$ rich Al-Ni-Co at temperatures above $0.6 \mathrm{~T}_{\text {melt }}$. The mobile $\mathrm{Al}$ atoms were located inside HBS supertiles and in the centres of decagonal columnar clusters with eight $\mathrm{Al}$ and two $\mathrm{Ni}$ atoms per period (Fig. 1). All diffusion processes observed with pair potentials also occur with the newly developed EAM potentials. Additionally, mobile $\mathrm{Al}$ atoms are found also in clusters with seven $\mathrm{Al}$ and three $\mathrm{Ni}$ atoms. Even the atoms in clusters with five $\mathrm{Al}$ and 
five Ni atoms, which were very stable with pair potentials, now contribute to the diffusion. $\mathrm{Ni}$ atoms in these clusters jump to neigbour positions, and nearby $\mathrm{Al}$ atoms fill the vacant sites. With pair potentials, transition metal (TM) atoms were practically immobile. The mobility of the TM atoms depends on the geometry of the site, but is independent of the chemical indentity. Compared to pair potentials, the additional diffusion processes lead to $\mathrm{Al}$ diffusivities which are by a factor 1.6 higher in the decagonal plane. In the periodic direction, there is almost no difference, since the same diffusion channels [1] dominate. The increase of the in-plane contribution makes the $\mathrm{Al}$ diffusion more isotropic. In contrast to the simulations with pair potentials, with EAM potentials the diffusivities for $\mathrm{Ni}$ are high enough to be determined quantitatively. The diffusion processes in Co-rich Al-Ni-Co and in $\mathrm{Al}-\mathrm{Cu}-\mathrm{Co}$ are similar, but have not been analysed in detail yet. All three decagonal structures have in common, that there are many sites which are large enough to accomodate more than one atom for a short time. Most of the time, they are singly occupied, but for short time periods they can be doubly occupied or empty. Most of the observed long range diffusion runs via such sites. In simulations with relatively simple crystalline alloys $\left(\mathrm{Al}_{3} \mathrm{Ni}\right.$, $\mathrm{Al}_{7} \mathrm{CoCu}_{2}$ ), all sites remain singly occupied all the time, so that these diffusion mechanisms cannot work there.

Time-averaged atom density maps calculated with ab-initio MD as well as classical MD with both EAM and pair potentials look very similar (Fig. 2). All TM sites and some Al sites remain fairly sharp, whereas most of the other $\mathrm{Al}$ sites are smeared out in certain directions, but are still stable and well indentifiable. With ab-initio MD and with EAM potentials, all spots are somewhat smoother than with pair potentials. This is especially so for the (dark) TM sites, which are very sharp with pair potentials. Hence, for TM atoms the EAM potentials are apparently much softer, and reproduce ab-initio result significantly better. The softer potential may also explain the higher TM atom diffusivities with EAM potentials.

Insert Fig. 2 about here, in the following arrangement:

Fig. 2a Fig. 2b Fig. 2c $\quad$ Fig. 2d $\quad$ Fig. 2e $\quad$ Fig. 2f

The energy barriers of the observed diffusion processes are determined als follows. The initial and final configurations are relaxed, and for each jumping atom ten equidistant positions between the initial and final configurations are calculated. The jumping atoms are then held fixed at these intermediate positions, while the positions of the others are relaxed again. For the configurations so obtained, the energies are computed, both abinitio and with EAM potentials. The energy barriers of a few example processes with three jumping atoms, either three $\mathrm{Al}$ or two $\mathrm{Al}$ and one $\mathrm{TM}$, are shown in Fig. 3. In these and many other examples that have been measured, the energy barriers determined ab-initio and with EAM potentials are quite similar. For jumps involving three $\mathrm{Al}$ atoms, they are in the range $0.5-2 \mathrm{eV}$, and for jumps with two $\mathrm{Al}$ and one $\mathrm{Ni}$ or $\mathrm{Cu}$ atom in the range $2-5 \mathrm{eV}$. Unfortunately, processes involving the correlated motion of a larger number of atoms are not easily detectable, so that it remains unclear whether there are still other relevant diffusion processes. 


\section{Insert Fig. 3 about here}

To determine the diffusion quantitatively, the mean square displacement (MSQD) of each atom type was measured as a function of time, for different temperatures and pressures. From the slopes of these curves, the diffusion constant $D$ was extracted and fitted to the usual Arrhenius law

$$
D=D_{0} e^{-\left(\frac{\Delta H+p \Delta V}{k_{B} T}\right)},
$$

from which the activation enthalpy $\Delta H$, activation volume $\Delta V$, and pre-exponential factor $D_{0}$ was determined, separately for the $x$-, the $y$-, and the $z$-directions. The values are given in Table 1. All diffusion measurements were carried out with samples of about 3000 atoms in six double layers, and ran over $2 \mathrm{~ns}$ (2 million time steps).

Insert Table 1 about here

The $\mathrm{Al}$ activation enthalpies are rather low, compared with the value for vacancy diffusion in $\mathrm{FCC} \mathrm{Al}(1.26 \mathrm{eV})$ [13]. The same holds true for the $\mathrm{Ni}$ activation enthalpies, which can be compared with the values measured in $\mathrm{Al}_{72.2} \mathrm{Ni}_{10.5} \mathrm{Co}_{16.9}\left(\Delta H_{x y}=2.62 \mathrm{eV}\right.$, $\Delta H_{z}=2.49 \mathrm{eV}$ ) [14]. However, due to the short time scales in the simulation, the concentration of vacancies or other diffusion vehicles may not be in equilibrium, so that only the migration enthalpy is measured [15], not the full activation enthalpy. The same problem occurs with the activation volumes, which are in the range of $10-50 \%$ of the average atomic volume, $\Omega$. These should be interpreted as migration volumes, not full activation volumes. The determined energy barriers compare better with the (expected) full activation enthalpies. There are possibly still other processes with lower activation enthalpy at work. Especially those involving a larger number of atoms are difficult to detect and analyse.

\section{Conclusion}

We have shown that MD simulations are suitable to determine diffusion in quasicrystals quantitatively, which in case of aluminium is not possible by experiment. The newly developed EAM potentials reproduce the ab-initio dynamics much better than previously used pair potentials [1], and allow to reliably determine energy barriers and atom density maps. Unfortunately, due to the short accessible time scales the measured activation enthalphies and activation volumes comprise only their migration part, not their formation part.

\section{Acknowledgement}

This work was funded by Deutsche Forschungsgemeinschaft under grant TR 154/23-1. 


\section{References}

[1] S. Hocker, F. Gähler, Phys. Rev. Lett. 93, 075901 (2004).

[2] F. Ercolessi, M. Parrinello, E. Tosatti, Phil. Mag. A 58, 213-226 (1988).

[3] M.S. Daw, M.I. Baskes, Phys. Rev. B 29, 6443-6453 (1984).

[4] F. Ercolessi, J.B. Adams, Europhys. Lett. 26 583-588 (1994).

[5] G. Kresse, J. Hafner, Phys. Rev. B 47, 558 (1993).

[6] G. Kresse, J. Furthmüller, Phys. Rev. B 54, 11169 (1996).

[7] G. Kresse, J. Furthmüller, Comp. Mat. Sci. 6, 15 (1996).

[8] G. Kresse, D. Joubert, Phys. Rev. B 59, 1758-1775 (1999).

[9] P. Brommer, F. Gähler, Effective potentials for quasicrystals from ab-initio data, preprint, submitted for the proceedings of ICQ9, 2005.

[10] M. Mihalkovič, M. et al., Phys. Rev. B 65, 104205 (2002).

[11] E. Cockayne, M. Widom, Phys. Rev. Lett. 81, 598-601 (1998).

[12] M. Mihalkovič, M. Widom, Tile-decoration model of a Co-rich AlCoNi quasicrystal derived from the $W$-AlCoNi approximant, preprint, submitted for the proceedings of ICQ9, 2005. See also http://alloy.phys.cmu.edu.

[13] P. Erhart, in: Landolt-Börnstein, New Series III, Vol. 25, ed. H. Ullmaier, Springer, Berlin, 1990.

[14] H. Mehrer, R. Galler, J. Alloys Compd. 342, 296-301 (2002).

[15] S. Grabowski, K. Kadau, P. Entel, Phase Trans. 75265 (2002). 
Table 1: Activation enthalpies, activation volumes, and pre-exponential factors for diffusion in decagonal Al-Ni-Co.

\begin{tabular}{|c|c|c|c|c|c|c|c|}
\hline \multicolumn{2}{|c|}{$\overline{\text { Sample }}$} & Ni-rich & Ni-rich & Ni-rich & Co-rich & Co-rich & Co-rich \\
\hline \multicolumn{2}{|c|}{ Atom, potential } & $\mathrm{Al}, \mathrm{EAM}$ & $\mathrm{Ni}, \mathrm{EAM}$ & Al, pair & $\mathrm{Al}, \mathrm{EAM}$ & $\mathrm{Ni}, \mathrm{EAM}$ & Al, pair \\
\hline$\Delta H_{x}$ & {$[\mathrm{eV}]$} & 0.70 & 0.98 & 0.89 & 0.83 & 0.93 & 0.63 \\
\hline$\Delta H_{z}$ & {$[\mathrm{eV}]$} & 0.58 & 1.11 & 0.64 & 0.83 & 0.97 & 0.66 \\
\hline$\Delta V_{x}$ & {$[\Omega]$} & 0.11 & 0.17 & 0.17 & 0.14 & - & 0.24 \\
\hline$\Delta V_{z}$ & {$[\Omega]$} & 0.11 & 0.27 & 0.28 & 0.14 & - & 0.33 \\
\hline$D_{0 x}$ & {$\left[\mathrm{~m}^{2} / \mathrm{s}\right]$} & $6.5 \times 10^{-8}$ & $5.1 \times 10^{-8}$ & $1.8 \times 10^{-7}$ & $1.2 \times 10^{-7}$ & $1.8 \times 10^{-8}$ & $3.1 \times 10^{-8}$ \\
\hline$D_{0 z}$ & {$\left[\mathrm{~m}^{2} / \mathrm{s}\right]$} & $3.5 \times 10^{-8}$ & $1.4 \times 10^{-7}$ & $6.3 \times 10^{-8}$ & $2.4 \times 10^{-7}$ & $1.6 \times 10^{-8}$ & $1.6 \times 10^{-7}$ \\
\hline
\end{tabular}

Figure 1: Diffusion processes in the decagonal plane (Al-Ni-Co, Ni-rich). Dark grey, large: Ni; light grey, large: Co; dark grey, small: Al initial positions; light grey, small: Al positions after 100ps. Initial and final positions are connected. Dashed lines mark supertiles. Dotted circles mark three types of decagonal clusters, differing in the Ni content.

Figure 2: Atom density maps of a supertile hexagon for Al-Ni-Co (left triple) and Al-Cu-Co (right triple). For each system, results with EAM potentials (left), ab-initio MD (middle), and pair potentials (right) are given.

Figure 3: Energy barriers of several diffusion processes with 3 atoms. 


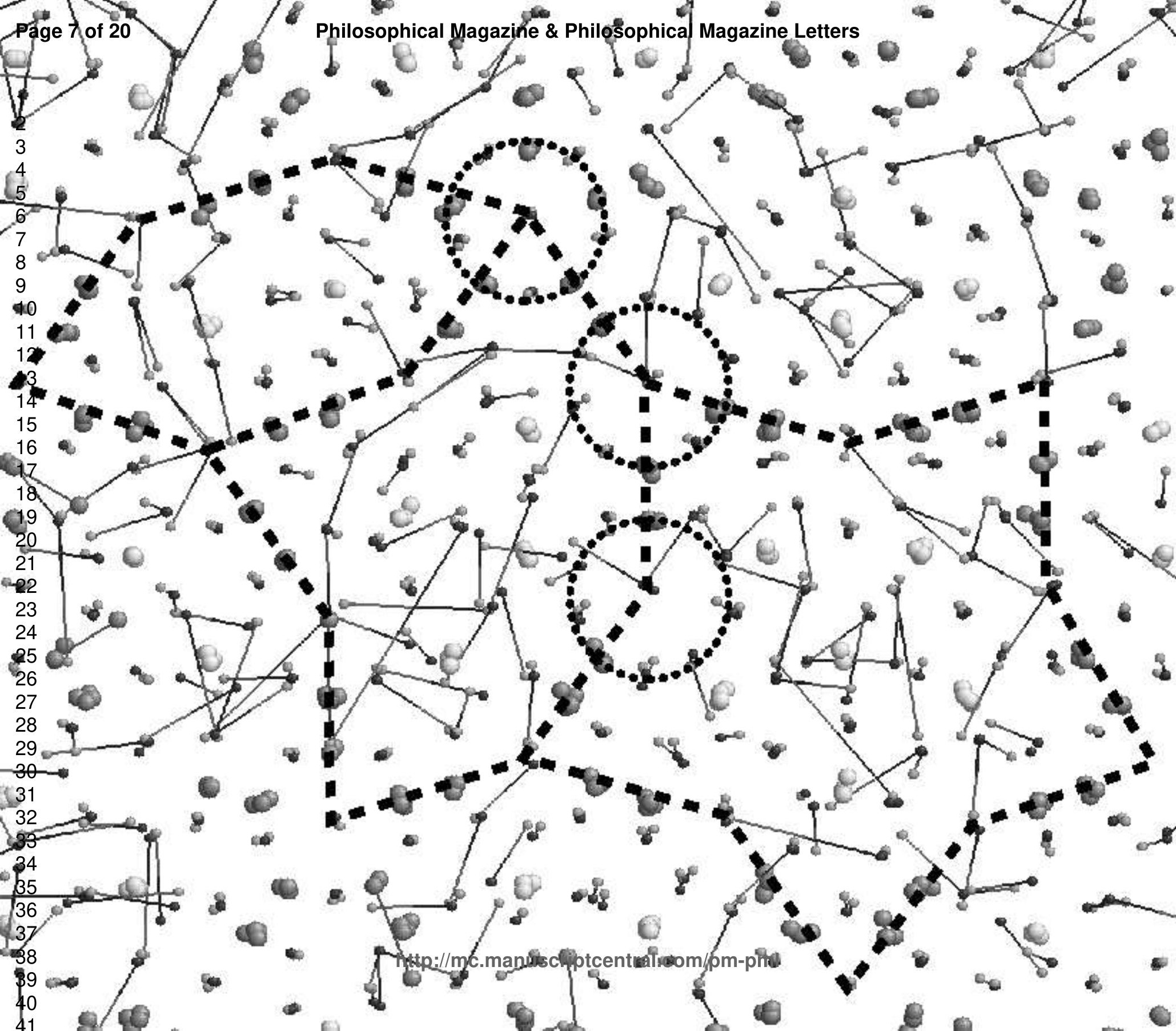




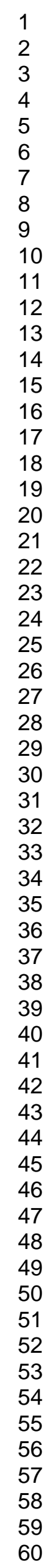




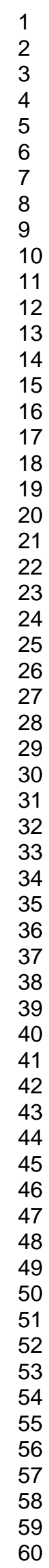

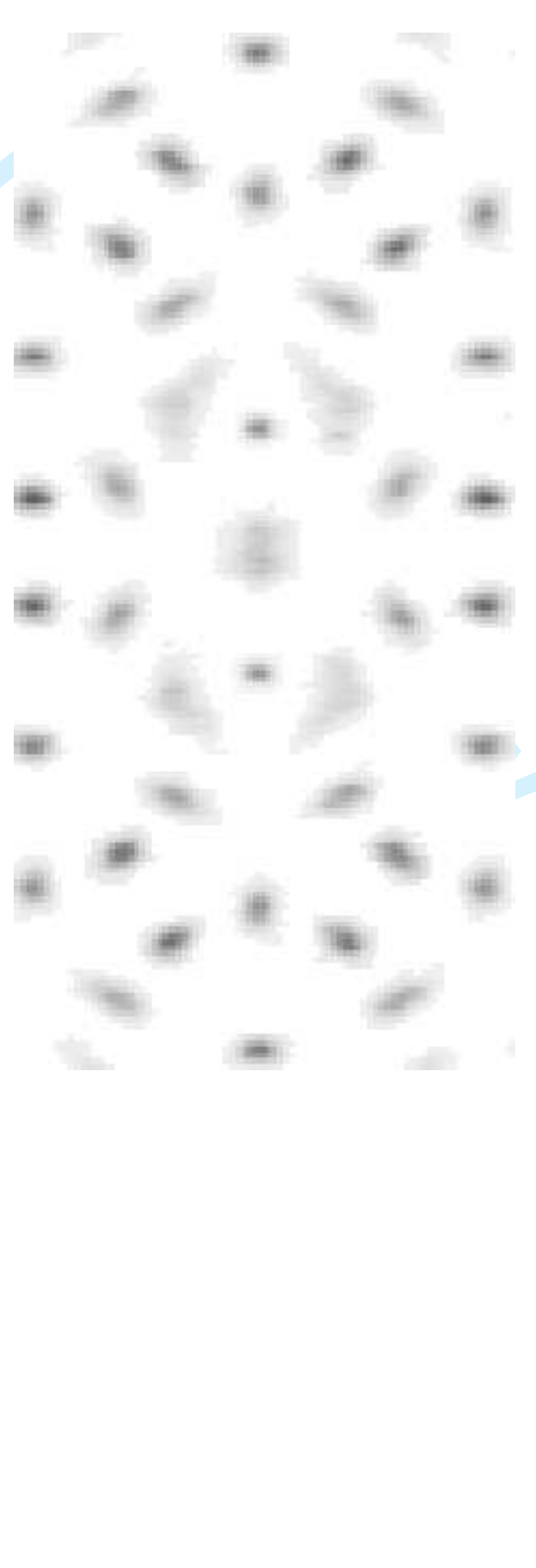

http://mc.manuscriptcentral.com/pm-pml 


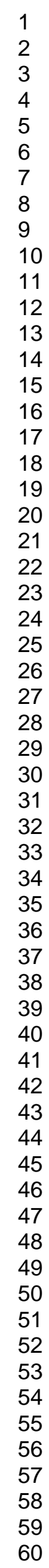




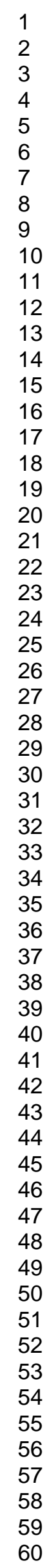

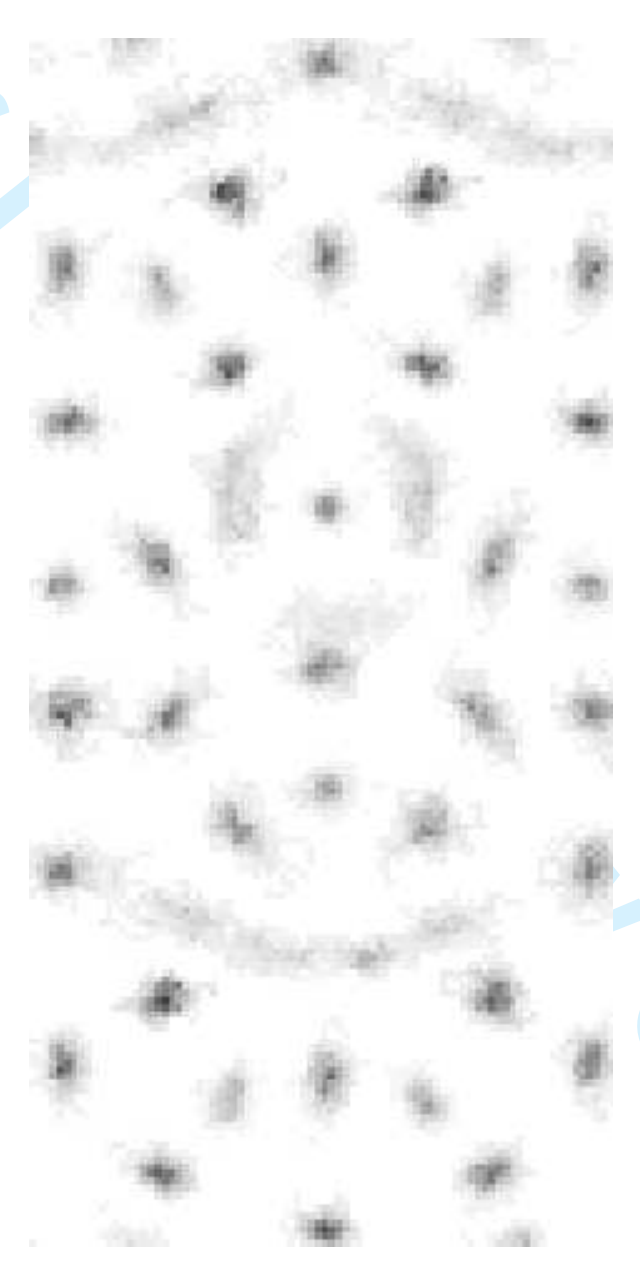

http://mc.manuscriptcentral.com/pm-pml 


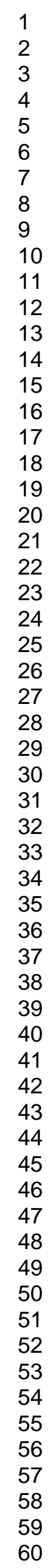

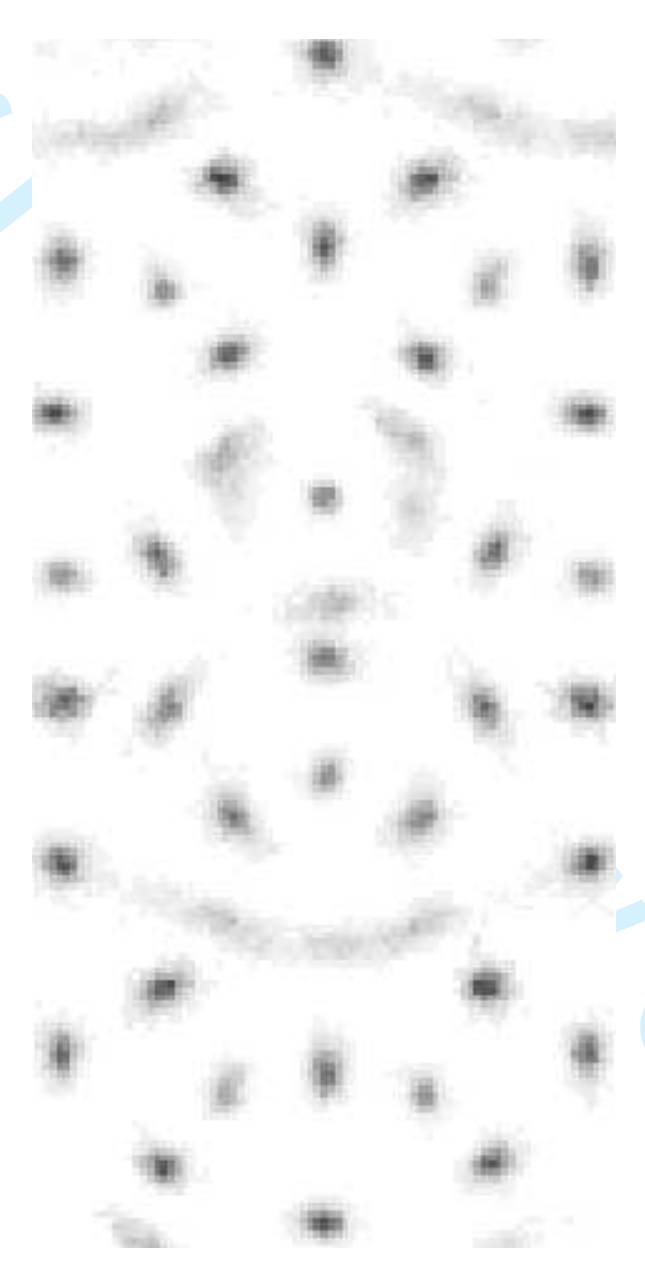

http://mc.manuscriptcentral.com/pm-pml 


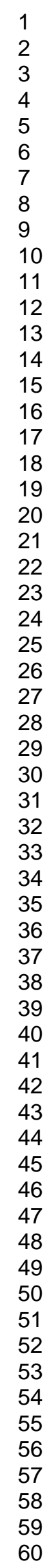

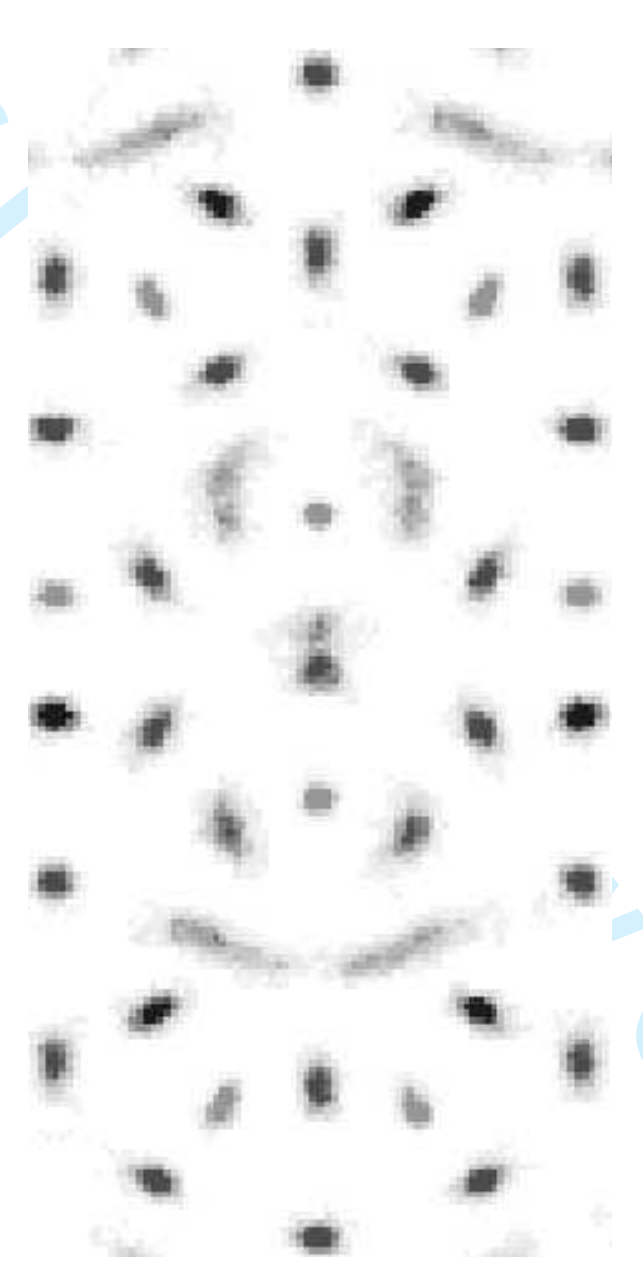

http://mc.manuscriptcentral.com/pm-pml 


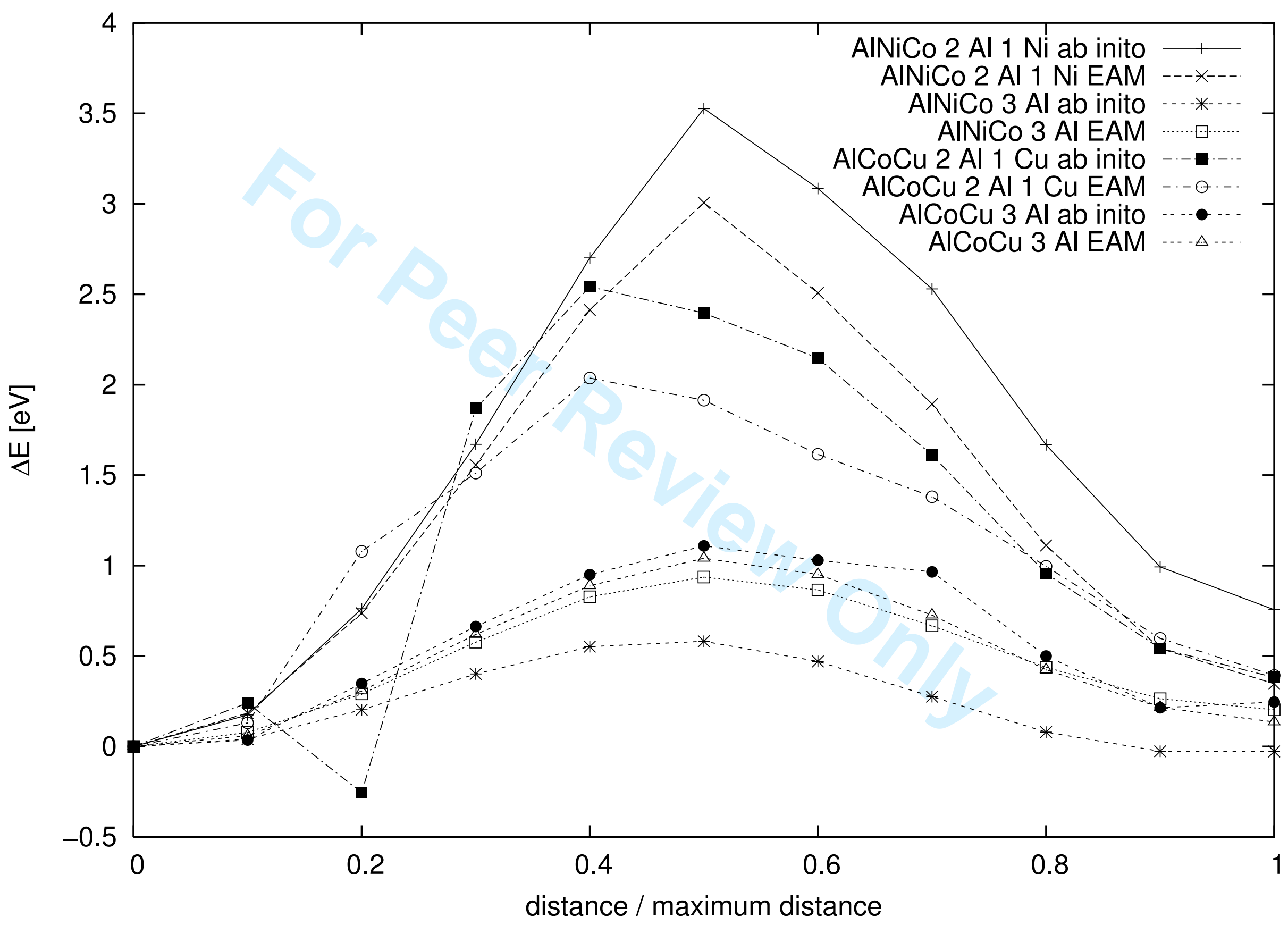




\title{
Molecular dynamics simulation of aluminium diffusion in decagonal quasicrystals
}

\author{
Stephen Hocker, Franz Gähler, and Peter Brommer \\ Institut für Theoretische und Angewandte Physik \\ Universität Stuttgart
}

June 24, 2010

\begin{abstract}
Aluminium diffusion in decagonal $\mathrm{Al}-\mathrm{Ni}-\mathrm{Co}$ and $\mathrm{Al}-\mathrm{Cu}-\mathrm{Co}$ quasicrystals is investigated by molecular dynamics simulations. Results obtained with newly developed EAM potentials are compared to previous work with effective pair potentials [Phys. Rev. Lett. 93, 075901 (2004)]. With both types of potential, strong aluminium diffusion is observed above two thirds of the melting temperature, and the general behaviour of the system is quite similar. The diffusion constant is measured as a function of temperature and pressure, and the activation enthalpies and activation volumes are determined from the resulting Arrhenius plot. For a number of important diffusion processes, the energy barriers are determined with molecular statics simulations. The qualitative behaviour of the dynamics is also confirmed by ab-initio simulations.
\end{abstract}

Keywords: Diffusion; Molecular dynamics simulation; Quasicrystals

\section{Introduction}

Atomic diffusion in quasicrystals is essential for many physical processes in these complex alloys. It is required for the formation of the equilibrium phase during high temperature annealing and for the motion of dislocations and other defects. Unfortunately, due to the lack of suitable radiotracers it is very hard to measure $\mathrm{Al}$ diffusion experimentally. No such studies have been presented so far. In a recent paper [1] we have shown that molecular dynamics (MD) simulations can complement the experimental methods. Molecular dynamics not only allows to determine the microscopic dynamics of the $\mathrm{Al}$ atoms, but also to measure the macroscopic diffusion constant as a function of temperature and pressure. MD simulations thus provide an excellent tool for the study of atomic dynamics both at the microscopic and the macroscopic level.

The quality of MD simulations crucially depends on the potentials. As quantummechanical simulations are limited to a few hundred atoms, which can neither provide 
enough statistics nor model the aperiodic nature of quasicrystals, classical effective potentials have to be used [1]. Since central pair potentials show some defiencies [2] in the description of metals and intermetallic compounds, we choose so-called Embedded Atom Method (EAM) [3] or glue potentials [2], which are better suited for metals. These include an additional embedding term, taking the form

$$
V=\frac{1}{2} \sum_{i \neq j} \Phi_{i j}\left(r_{i j}\right)+\sum_{i} U_{i}\left(n_{i}\right), \quad n_{i}=\sum_{i \neq j} \rho_{j}\left(r_{i j}\right),
$$

where $U_{i}$ describes the energy of embedding atom $i$ in a density $n_{i}$, which is the sum of contributions $\rho_{j}$ from neighbours $j$ at distances $r_{i j}$. We developed EAM potentials for Al-Ni-Co and Al-Cu-Co using the Force Matching Method of Ercolessi and Adams [4]. For a selection of small reference configurations the cohesion energies, stresses and forces on individual atoms are calculated by quantum-mechanical ab-initio methods. All ab-initio calculations in this work are carried out with VASP [5-7], using the Projector Augmented Wave (PAW) method [8]. The potential parameters are then fitted to optimally reproduce these data, using a least square fit. Details on the potential developement are given in [9]. The capabilities of the obtained potentials crucially depend on the selection of reference configurations. For the present purpose (diffusion simulations), high temperature structures are given a large weight in the collection of reference configurations, so that the potentials are trained to describe high temperature processes well.

Three different model structures are considered. The Ni-rich structure of decagonal Al$\mathrm{Ni}$-Co (composition $\mathrm{Al}_{72} \mathrm{Ni}_{21} \mathrm{Co}_{9}$ ) and the structure of decagonal Al-Cu-Co (composition $\mathrm{Al}_{70} \mathrm{Cu}_{10} \mathrm{Co}_{20}$ ) both consist of an alternating stacking of two different layers decorating the same hexagon-boat-star (HBS) tiling, which results in a period of about $4 \AA[10,11]$. We use sligthly modified variants determined in relaxation simulations, where it was found that the two innermost $\mathrm{Al}$ atoms in the star tiles prefer different positions, and also break the $4 \AA$ periodicity locally to an $8 \AA$ periodicity [1]. The model structure for Al-Cu-Co is obtained from the Ni-rich decoration by replacing each $\mathrm{Ni}-\mathrm{Ni}$ pair on supertile edges by a $\mathrm{Cu}$-Co pair. Lacking a detailed quasiperiodic model of the Co-rich phase, simulations of this structure were performed with a periodic approximant [12]. Its structure differs significantly from the Co-rich sample used in [1].

Insert Fig. 1 about here

\section{Results}

In previous work [1] with pair potentials, significant diffusion of $\mathrm{Al}$ was observed in $\mathrm{Ni}$ rich $\mathrm{Al}-\mathrm{Ni}$-Co at temperatures above $0.6 \mathrm{~T}_{\text {melt }}$. The mobile $\mathrm{Al}$ atoms were located inside HBS supertiles and in the centres of decagonal columnar clusters with eight $\mathrm{Al}$ and two Ni atoms per period (Fig. 1). All diffusion processes observed with pair potentials also occur with the newly developed EAM potentials. Additionally, mobile Al atoms are found also in clusters with seven $\mathrm{Al}$ and three $\mathrm{Ni}$ atoms. Even the atoms in clusters with five $\mathrm{Al}$ and 
five $\mathrm{Ni}$ atoms, which were very stable with pair potentials, now contribute to the diffusion. $\mathrm{Ni}$ atoms in these clusters jump to neigbour positions, and nearby $\mathrm{Al}$ atoms fill the vacant sites. With pair potentials, transition metal (TM) atoms were practically immobile. The mobility of the TM atoms depends on the geometry of the site, but is independent of the chemical indentity. Compared to pair potentials, the additional diffusion processes lead to $\mathrm{Al}$ diffusivities which are by a factor 1.6 higher in the decagonal plane. In the periodic direction, there is almost no difference, since the same diffusion channels [1] dominate. The increase of the in-plane contribution makes the $\mathrm{Al}$ diffusion more isotropic. In contrast to the simulations with pair potentials, with EAM potentials the diffusivities for $\mathrm{Ni}$ are high enough to be determined quantitatively. The diffusion processes in Co-rich Al-Ni-Co and in Al-Cu-Co are similar, but have not been analysed in detail yet. All three decagonal structures have in common, that there are many sites which are large enough to accomodate more than one atom for a short time. Most of the time, they are singly occupied, but for short time periods they can be doubly occupied or empty. Most of the observed long range diffusion runs via such sites. In simulations with relatively simple crystalline alloys $\left(\mathrm{Al}_{3} \mathrm{Ni}\right.$, $\mathrm{Al}_{7} \mathrm{CoCu}_{2}$ ), all sites remain singly occupied all the time, so that these diffusion mechanisms cannot work there.

Time-averaged atom density maps calculated with ab-initio MD as well as classical MD with both EAM and pair potentials look very similar (Fig. 2). All TM sites and some Al sites remain fairly sharp, whereas most of the other $\mathrm{Al}$ sites are smeared out in certain directions, but are still stable and well indentifiable. With ab-initio MD and with EAM potentials, all spots are somewhat smoother than with pair potentials. This is especially so for the (dark) TM sites, which are very sharp with pair potentials. Hence, for TM atoms the EAM potentials are apparently much softer, and reproduce ab-initio result significantly better. The softer potential may also explain the higher TM atom diffusivities with EAM potentials.

Insert Fig. 2 about here, in the following arrangement:

Fig. 2a Fig. 2b Fig. 2c $\quad$ Fig. 2d $\quad$ Fig. 2e $\quad$ Fig. 2f

The energy barriers of the observed diffusion processes are determined als follows. The initial and final configurations are relaxed, and for each jumping atom ten equidistant positions between the initial and final configurations are calculated. The jumping atoms are then held fixed at these intermediate positions, while the positions of the others are relaxed again. For the configurations so obtained, the energies are computed, both abinitio and with EAM potentials. The energy barriers of a few example processes with three jumping atoms, either three $\mathrm{Al}$ or two $\mathrm{Al}$ and one $\mathrm{TM}$, are shown in Fig. 3. In these and many other examples that have been measured, the energy barriers determined ab-initio and with EAM potentials are quite similar. For jumps involving three $\mathrm{Al}$ atoms, they are in the range $0.5-2 \mathrm{eV}$, and for jumps with two $\mathrm{Al}$ and one $\mathrm{Ni}$ or $\mathrm{Cu}$ atom in the range $2-5 \mathrm{eV}$. Unfortunately, processes involving the correlated motion of a larger number of atoms are not easily detectable, so that it remains unclear whether there are still other relevant diffusion processes. 


\section{Insert Fig. 3 about here}

To determine the diffusion quantitatively, the mean square displacement (MSQD) of each atom type was measured as a function of time, for different temperatures and pressures. From the slopes of these curves, the diffusion constant $D$ was extracted and fitted to the usual Arrhenius law

$$
D=D_{0} e^{-\left(\frac{\Delta H+p \Delta V}{k_{B} T}\right)},
$$

from which the activation enthalpy $\Delta H$, activation volume $\Delta V$, and pre-exponential factor $D_{0}$ was determined, separately for the $x$-, the $y$-, and the $z$-directions. The values are given in Table 1. All diffusion measurements were carried out with samples of about 3000 atoms in six double layers, and ran over 2 ns (2 million time steps).

Insert Table 1 about here

The $\mathrm{Al}$ activation enthalpies are rather low, compared with the value for vacancy diffusion in $\mathrm{FCC} \mathrm{Al}(1.26 \mathrm{eV})$ [13]. The same holds true for the $\mathrm{Ni}$ activation enthalpies, which can be compared with the values measured in $\mathrm{Al}_{72.2} \mathrm{Ni}_{10.5} \mathrm{Co}_{16.9}\left(\Delta H_{x y}=2.62 \mathrm{eV}\right.$, $\Delta H_{z}=2.49 \mathrm{eV}$ ) [14]. However, due to the short time scales in the simulation, the concentration of vacancies or other diffusion vehicles may not be in equilibrium, so that only the migration enthalpy is measured [15], not the full activation enthalpy. The same problem occurs with the activation volumes, which are in the range of $10-50 \%$ of the average atomic volume, $\Omega$. These should be interpreted as migration volumes, not full activation volumes. The determined energy barriers compare better with the (expected) full activation enthalpies. There are possibly still other processes with lower activation enthalpy at work. Especially those involving a larger number of atoms are difficult to detect and analyse.

\section{Conclusion}

We have shown that MD simulations are suitable to determine diffusion in quasicrystals quantitatively, which in case of aluminium is not possible by experiment. The newly developed EAM potentials reproduce the ab-initio dynamics much better than previously used pair potentials [1], and allow to reliably determine energy barriers and atom density maps. Unfortunately, due to the short accessible time scales the measured activation enthalphies and activation volumes comprise only their migration part, not their formation part.

\section{Acknowledgement}

This work was funded by Deutsche Forschungsgemeinschaft under grant TR 154/23-1. 


\section{References}

[1] S. Hocker, F. Gähler, Phys. Rev. Lett. 93, 075901 (2004).

[2] F. Ercolessi, M. Parrinello, E. Tosatti, Phil. Mag. A 58, 213-226 (1988).

[3] M.S. Daw, M.I. Baskes, Phys. Rev. B 29, 6443-6453 (1984).

[4] F. Ercolessi, J.B. Adams, Europhys. Lett. 26 583-588 (1994).

[5] G. Kresse, J. Hafner, Phys. Rev. B 47, 558 (1993).

[6] G. Kresse, J. Furthmüller, Phys. Rev. B 54, 11169 (1996).

[7] G. Kresse, J. Furthmüller, Comp. Mat. Sci. 6, 15 (1996).

[8] G. Kresse, D. Joubert, Phys. Rev. B 59, 1758-1775 (1999).

[9] P. Brommer, F. Gähler, Effective potentials for quasicrystals from ab-initio data, preprint, submitted for the proceedings of ICQ9, 2005.

[10] M. Mihalkovič, M. et al., Phys. Rev. B 65, 104205 (2002).

[11] E. Cockayne, M. Widom, Phys. Rev. Lett. 81, 598-601 (1998).

[12] M. Mihalkovič, M. Widom, Tile-decoration model of a Co-rich AlCoNi quasicrystal derived from the $W$-AlCoNi approximant, preprint, submitted for the proceedings of ICQ9, 2005. See also http://alloy.phys.cmu.edu.

[13] P. Erhart, in: Landolt-Börnstein, New Series III, Vol. 25, ed. H. Ullmaier, Springer, Berlin, 1990.

[14] H. Mehrer, R. Galler, J. Alloys Compd. 342, 296-301 (2002).

[15] S. Grabowski, K. Kadau, P. Entel, Phase Trans. 75265 (2002). 
Table 1: Activation enthalpies, activation volumes, and pre-exponential factors for diffusion in decagonal Al-Ni-Co.

\begin{tabular}{lccccccc}
\hline \multicolumn{2}{l}{$\begin{array}{l}\text { Sample } \\
\text { Atom, potential }\end{array}$} & $\begin{array}{c}\text { Ni-rich } \\
\text { Al, EAM }\end{array}$ & $\begin{array}{c}\text { Ni-rich } \\
\text { Ni, EAM }\end{array}$ & $\begin{array}{c}\text { Ni-rich } \\
\text { Al, pair }\end{array}$ & $\begin{array}{c}\text { Co-rich } \\
\text { Al, EAM }\end{array}$ & $\begin{array}{c}\text { Co-rich } \\
\text { Ni, EAM }\end{array}$ & $\begin{array}{c}\text { Co-rich } \\
\text { Al, pair }\end{array}$ \\
\hline$\Delta H_{x}$ & {$[\mathrm{eV}]$} & 0.70 & 0.98 & 0.89 & 0.83 & 0.93 & 0.63 \\
$\Delta H_{z}$ & {$[\mathrm{eV}]$} & 0.58 & 1.11 & 0.64 & 0.83 & 0.97 & 0.66 \\
$\Delta V_{x}$ & {$[\Omega]$} & 0.11 & 0.17 & 0.17 & 0.14 & - & 0.24 \\
$\Delta V_{z}$ & {$[\Omega]$} & 0.11 & 0.27 & 0.28 & 0.14 & - & 0.33 \\
$D_{0 x}$ & {$\left[\mathrm{~m}^{2} / \mathrm{s}\right]$} & $6.5 \times 10^{-8}$ & $5.1 \times 10^{-8}$ & $1.8 \times 10^{-7}$ & $1.2 \times 10^{-7}$ & $1.8 \times 10^{-8}$ & $3.1 \times 10^{-8}$ \\
$D_{0 z}$ & {$\left[\mathrm{~m}^{2} / \mathrm{s}\right]$} & $3.5 \times 10^{-8}$ & $1.4 \times 10^{-7}$ & $6.3 \times 10^{-8}$ & $2.4 \times 10^{-7}$ & $1.6 \times 10^{-8}$ & $1.6 \times 10^{-7}$ \\
\hline
\end{tabular}

Figure 1: Diffusion processes in the decagonal plane (Al-Ni-Co, Ni-rich). Dark grey, large: Ni; light grey, large: Co; dark grey, small: Al initial positions; light grey, small: Al positions after 100ps. Initial and final positions are connected. Dashed lines mark supertiles. Dotted circles mark three types of decagonal clusters, differing in the Ni content.

Figure 2: Atom density maps of a supertile hexagon for Al-Ni-Co (left triple) and Al-Cu-Co (right triple). For each system, results with EAM potentials (left), ab-initio MD (middle), and pair potentials (right) are given.

Figure 3: Energy barriers of several diffusion processes with 3 atoms. 